\title{
Productos de precipitación radar en modelos hidrometeorológicos no agregados
}

\author{
José Luis Cervantes Rodríguez ${ }^{1}$ (jcervantesr@aemet.es) \\ María Lorena Martínez Chenoll² (mamarche@alumni.upv.es) \\ Amparo Moreno Durá2 (demodu@hma.upv.es)
}

${ }^{1}$ AEMET / Delegación Territorial en la Comunidad Valenciana

${ }^{2}$ Universidad Politécnica de Valencia / Escuela Técnica Superior de Ingenieros de Caminos, Canales y Puertos /

Departamento de Ingeniería Hidráulica y Medio Ambiente

\begin{abstract}
RESUMEN
El enfoque hidrometeorológico en cuencas hidrográficas es indispensable para el pronóstico de avenidas en ausencia de series fiables de aforo; puesto que simula el proceso de transformación precipitación-escorrentía mediante modelos de mayor o menor complejidad, usando como input el dato pluviométrico. Los grandes avances alcanzados por los modelos hidrometeorológicos distribuidos y pseudodistribuidos (no agregados) han permitido a los hidrólogos no solo aumentar las capacidades para simular crecidas en tiempo real en todos los puntos de la cuenca (corto-medio plazo), sino desarrollar herramientas de predicción de recursos hídricos (predicción estacional) y evaluar más certeramente los efectos del cambio climático (proyección hidrológica).

Estos modelos exigen que las distribuciones espaciales y temporales del campo de precipitación, y sus variabilidades internas se describan con resoluciones espaciales inferiores al $\mathrm{km}^{2} \mathrm{y}$ temporales que van de minutos a años. Frente a la caracterización clásica del campo de precipitación basada en datos de estaciones pluviométricas distribuidas de forma discreta y heterogénea por el territorio; caracterización que construye hietogramas de diseño únicos para toda la cuenca y que usa interpolación y promedio superficial, los productos de precipitación obtenidos de las observaciones radar satisfacen de manera natural estas exigencias.
\end{abstract}

Cae entonces del lado del meteorólogo/climatólogo la necesidad de explotar los productos de precipitación radar para que puedan amoldarse a estos modelos hidrológicos de última generación. Mediante la elección de una cuenca de estudio, se ilustrará el proceso completo.

PALABRAS CLAVE: avenidas; modelos hidrometeorológicos; campo de precipitación; productos radar; predicción estacional; proyección hidrológica. 
